\title{
The 2D Finite Difference Numerical Modelling of P-SV Wave Propagation In Elastic Heterogeneous Medium Using Graphic Processing Unit: Case Study of Mount Merapi Topography, Yogyakarta
}

\author{
Theodorus Permana ${ }^{1}$, Sudarmaji ${ }^{2}$ \\ Geophysics Sub department, Department of Physics, \\ Faculty of Mathematics and Natural Sciences, Universitas Gadjah Mada \\ Yogyakarta, Indonesia \\ E-mail: ${ }^{1}$ theodorus.permana@gmail.com, ${ }^{2}$ ajisaroji@ugm.ac.id
}

\begin{abstract}
Simulation of seismic wave propagation has been very important way to study the relation between a specific condition of earth medium and seismogram recorded during a seismic event such as an earthquake. In this study, a numerical modelling of P-SV wave propagation in elastic heterogeneous medium has been done using fourth order finite-difference method. The medium model was created based on topography profile of selected line intersecting Mount Merapi, where 200 meters thick low-velocity layer has been added. The computation was performed using an NVIDIA CUDA-enabled graphics card. Synthetic seismogram was recorded at three receiver stations and was obtained by using a deep seismic source and a shallow seismic source located within the volcano. Results shows significant differences of wave propagation in presence of topography and low-velocity layer, while compared to the homogeneous version of the model which excludes the low-velocity layer. The formation of surface wave is also proved to significantly affect the recorded seismogram. Topography, low-velocity layer located at near-surface, and generated surface wave are suspected to be responsible for the complexity of the recorded seismogram. Compared to computation by using only CPU, usage of NVIDIA graphics processing unit proved to be successful to accelerate the execution time by 23 times.
\end{abstract}

Keywords: finite-difference, GPU, mount Merapi, numerical modelling, seismic wave propagation

\section{INTRODUCTION}

Finite-difference has been recognized as a relatively simple method to simulate seismic wave propagation within the earth, by solving the wave equation using differential method, or known as strong form. Finite-difference requires the propagation medium to be discretized into rectangular grid. However, the rectangular grid cannot accurately represent the topography of earth surface as free-surface boundary. To handle this problem, Robertsson [1] proposed a method to approach this problem of free-surface, which developed from [2]. For other medium boundaries, the convolutional perfectly matched layer (CPML) has been used as absorbing boundary condition, which has been proved effective to absorb wave energy in grid boundaries [3].

This simulation was performed using an NVIDIA graphics processing unit (GPU), which is publicly available for daily use. Today GPU technology has allowed us to perform various physics simulation, such as seismic wave propagation as in [4] and acoustics simulation as in [5] with faster calculation in shorter execution time than only using the computer's CPU. In this paper, the term "using GPU" means that GPU is used in addition to CPU, where the calculation will be mainly performed by GPU.

In order to perform seismic wave propagation simulation, a computer program written in $\mathrm{C}++$ language has been created to solve seismic wave equation using finitedifference in staggered-grid scheme [2,7,8,9], with CPML implementation based on [3], and using method by [1] to model wave propagation in irregular earth surface.

The propagation medium is a two-layers, elastic medium with irregular upper boundary based on topographical profile from selected line intersecting the summit of Mt. Merapi. Multi-layer medium is also represents the heterogeneity of the medium [10]. For this study, the term "heterogeneous" is used to referring to the two-layer medium.

Mt. Merapi, an active stratovolcano in the border of Yogyakarta and Central Java, was chosen in this study because of its relatively simple topography, although it is dynamically changed by eruption process which occurred regularly, since the volcano is recognized as one of most active and dangerous volcano [11]. High number of study which took interest on Mt. Merapi also made it easier to collect additional data to be used in this study, such as [12].

This study is designed to mimic real passive seismic survey, where ground displacements are recorded by seismometer. In this study, a "seismometer" is a point in the medium where ground displacement will be recorded. After synthetic seismogram from the simulation has been obtained, simple analysis are then performed to compare the seismogram resulted in different cases, such as source location and medium homogeneity, and also to prove the advantages of using GPU.

\section{THEORIES}

\section{A. Seismic Wave Equation}

Wave propagation within the earth are affected by elastic properties of rock. The theory of elasticity relates the applied force and the changes in medium shape or size, which expressed in terms of stress and strain. Based on second law 
of Newton, the unbalanced stress $\sigma$ applied to a cube's surface are equal to mass times acceleration. Thus we have the motion along $\mathrm{x}$-axis [13].

$$
\rho \frac{\partial^{2} u_{x}}{\partial t^{2}}=\frac{\partial \sigma_{x x}}{\partial x}+\frac{\partial \sigma_{x y}}{\partial y}+\frac{\partial \sigma_{x z}}{\partial z}
$$

where $\rho$ is medium density, $\sigma_{x x}$ is normal stress, $\sigma_{x y}$ and $\sigma_{x z}$ are shear stress components. The left-hand side in (1) contains temporal derivative and the right-hand side contains spatial derivatives of stress components. Equation for $\mathrm{y}$ and Z-axis can be derived using similar relation. Equation (1) relates the displacements $u$ to the stress, in more general form known as homogeneous wave motion [10].

To solve the equation in two-dimension, it will be evaluated in $\mathrm{x}$ and $\mathrm{z}$-direction. By expressing stress in strain, then expressing strain in displacement using Hooke's Law, calculating normal and shear stress components and then transformed it into first-order hyperbolic equation, one could obtain the velocity-stress equation set [8].

$$
\begin{gathered}
\rho \frac{\partial v_{x}}{\partial t}=\frac{\partial \sigma_{x x}}{\partial x}+\frac{\partial \sigma_{x z}}{\partial z} \\
\rho \frac{\partial v_{z}}{\partial t}=\frac{\partial \sigma_{x z}}{\partial x}+\frac{\partial \sigma_{z z}}{\partial z} \\
\frac{\partial \sigma_{x x}}{\partial t}=(\lambda+2 \mu) \frac{\partial v_{x}}{\partial x}+\frac{\partial v_{z}}{\partial z} \\
\frac{\partial \sigma_{z z}}{\partial t}=(\lambda+2 \mu) \frac{\partial v_{z}}{\partial z}+\frac{\partial v_{x}}{\partial x} \\
\frac{\partial \sigma_{x z}}{\partial t}=\mu\left(\frac{\partial v_{x}}{\partial z}+\frac{\partial v_{z}}{\partial x}\right),
\end{gathered}
$$

where $v_{x}$ and $v_{z}$ is particle velocity in $\mathrm{x}$ - and z-direction and $\mu$ is the shear modulus. $\lambda$ and $\mu$ are known as Lamé parameters, Numerical solution in (2) to (6) will be solved using fourth-order in space, second-order in time finitedifference approach [2].

\section{B. Staggered-Grid Finite-Difference}

Before simulation begins, medium parameters have to be discretized into a structured grid in 2D Cartesian system. In this simulation, staggered-grid scheme [7-9] is used, where each component of velocity and stress will be calculated in separated grid position. However, normal stresses will occupy the same position (Fig. 1).

Numerical solution for the fourth-order finite-difference introduced by [2] are similar to those of second-order scheme [7-9], except for calculation of velocity and stress components at a grid point will include four points closest to it (in $\mathrm{x}$ - and z-direction, respectively). Fig. 2 illustrate this scheme for calculating both velocity and stress components.

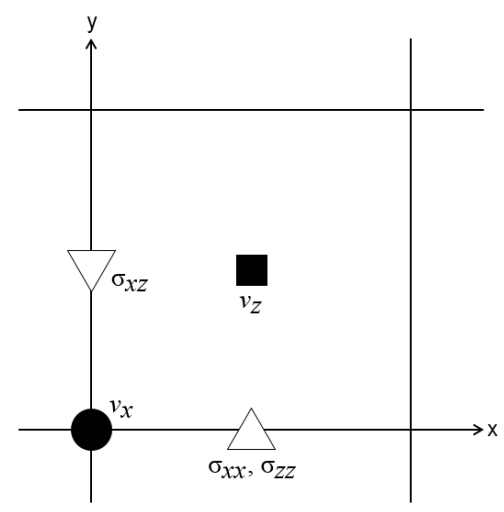

Figure 1. Staggered-grid scheme, showing different position of velocity and stress components in standard Cartesian system rectangular grid, based on [8].
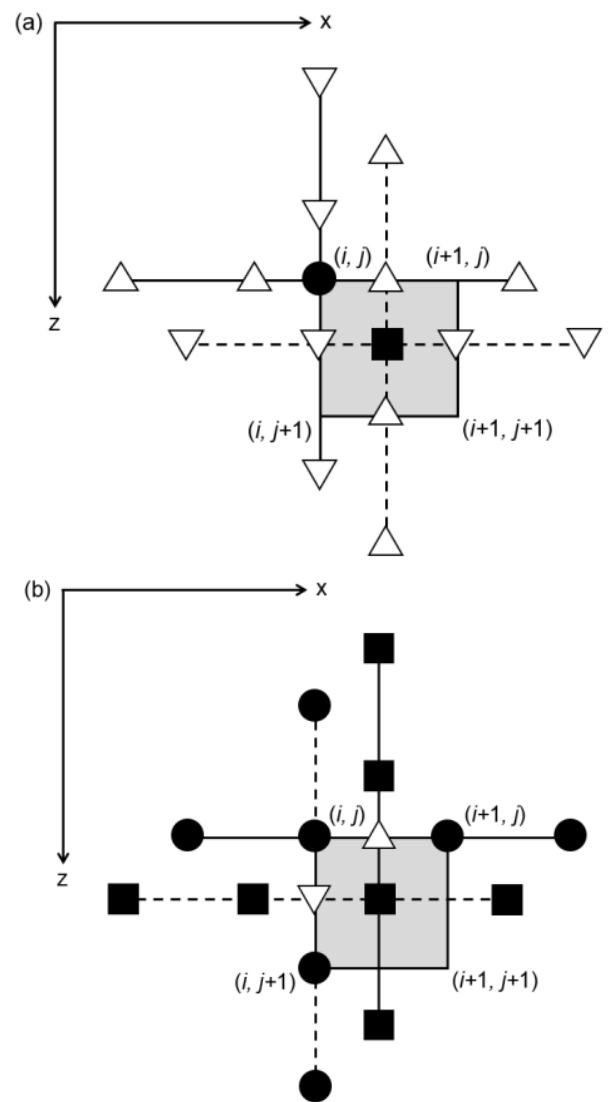

Figure 2. Staggered-grid stencils for fourth-order approximation based on [2], the gray square has area $h^{2}$, with $h$ is spatial distance, and the grid-point is located at index $(i, j)$. Notice that in finite-difference grid, $\mathrm{z}$-axis is positive downward: (a) spatial stencils for velocity update and (b) spatial stencils for stress update.

\section{Boundary Condition}

There are two types of boundary condition used in this simulation: absorbing boundary condition to represent an infinite medium, and free-surface condition at upper boundary of the medium, to model wave propagation in presence of surface topography (Fig. 3). For absorbing boundary, the CPML technique is used, based on [3]. This 
technique is a elastodynamic version from the general CPML form developed by [14], which is an improvement from the classic perfectly matched layer (PML) introduced by [15]. PML has been proven to be efficiently absorb wave energy along the medium boundary, preventing the wave from reflecting back to the computation domain.

In order to deal with surface topography, method described in [1] is used. The method is a quite robust method used to solve wave propagation in irregular free-surface, which represents earth surface. This method approximate the smooth earth surface as finite steps, classifying the gridpoints along free-surface into seven categories, with each category will be treated uniquely in order to calculate the velocity and stress components. The air layer above the freesurface will be treated as vacuum.

\section{Stability Analysis}

In explicit time scheme, the time step $\Delta t$ and spatial distance $\Delta x$ and $\Delta z$ must be specified in order to satisfy the stability condition [8]

$$
V_{p} \Delta t \sqrt{\frac{1}{\Delta x^{2}}+\frac{1}{\Delta z^{2}}} \leq 1 .
$$

For fourth-order finite-difference scheme, the following stability condition are used [2].

$$
\Delta t<\frac{\Delta x}{\sqrt{2 V_{p}\left(\frac{9}{8}+\frac{1}{24}\right)}} .
$$

where $V p$ is $\mathrm{P}$-wave velocity in the medium. The value $9 / 8$ and $1 / 24$ are coefficients of the fourth-order approximation. The value $\Delta t$ for fourth-order scheme (8) is smaller than second-order scheme (7).

Numerical dispersion analysis for fourth-order scheme shows that at least 5 grid-points/wavelength to minimize the effect of grid dispersion [2], while 10 grid-points/wavelength are needed for second-order scheme $[8,16]$. However, the accuracy will decreased along the topography, where at least 15 grid-points/wavelength are needed in the presence of topography [1]. This is caused by scattering due to the usage of finite steps to represents smooth boundary $[1,17]$.

\section{E. Graphics Processing Unit}

Computation using a GPU has been proven to successfully accelerate the execution time compared to traditional CPU. A CPU rely on small number of cores, with faster execution speed performed by each core. A GPU rely on vast number of cores with slower execution speed. However, if all cores capable to perform a calculation simultaneously, the usage of high number of cores will be an advantage. This concept has been known as parallel computation.

This study used NVIDIA GPU with CUDA technology, which enables NVIDIA graphics card to support multipurpose programming, beside its capability to process graphic pixel data. In order to perform multi-purpose programming using NVIDIA graphic card, an extension version of $\mathrm{C} / \mathrm{C}++$ language, known as $\mathrm{CUDA} \mathrm{C} / \mathrm{C}++$, is used [18].

A GPU hardware consist of a group of streaming multiprocessor (SM), each has a number of streaming processor (SP) which shares cache. Each SP consist of high number of thread, in which the computation will be executed. In this study, one grid-point of the medium is represented by one thread (Fig. 4).

Execution process initialized by assign blocks in the SM, each block can contain up to 1,024 threads. Although each SM can contain up to 8 blocks, a single calculation may require hundreds of blocks to execute. The system will create an execution list to control which blocks will be executed by the SM. During execution, a block is divided into warp, a group of 32 threads. Each SM can contain up to 1,536 threads.

From programming aspect, there is three basic operation while executing a kernel (a subroutine executed using GPU). First, parameter initialization and input data transfer from the

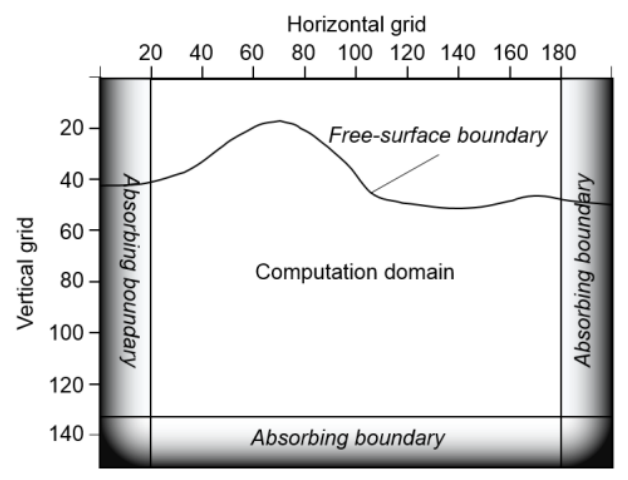

Figure 3. Boundary conditions used in this study to represent twodimensional infinite earth with presence of surface topography.

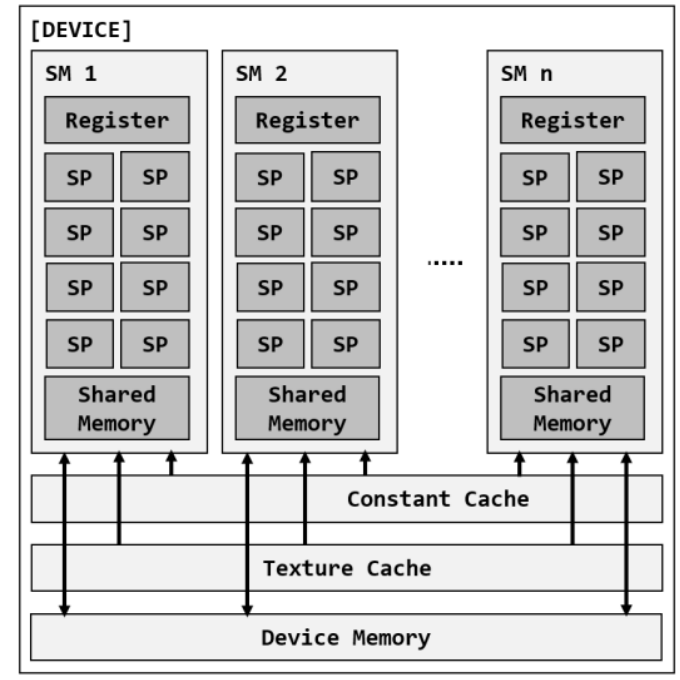

Figure 4. Illustration of cores grouping in a GPU device. SM is streaming multiprocessor, and SP is streaming processor. 
host (CPU) to the device (GPU) global memory. Second, the kernel are then executed n-times, which requires $n$-threads. After execution is completed, the results then transferred back to the host $[6,18]$.

\section{Methodology}

\section{A. Topography Profile of Mount Merapi}

Topography profile of Mount Merapi was obtained from ASTER GDEM global elevation data which has spatial resolution of 1.5 arc second. Selected line (Fig. 5), which intersecting the summit of Mount Merapi, has $32 \mathrm{~km}$ length, from which the elevation data will be extracted. The profile are then interpreted as upper free-surface boundary of the elastic medium. The air layer above the surface will be treated as vacuum.

There are three fictional stations located in the surface along the profile, in order to record the seismic wave in summit area (MRP), the slope (PLA), and relatively distant
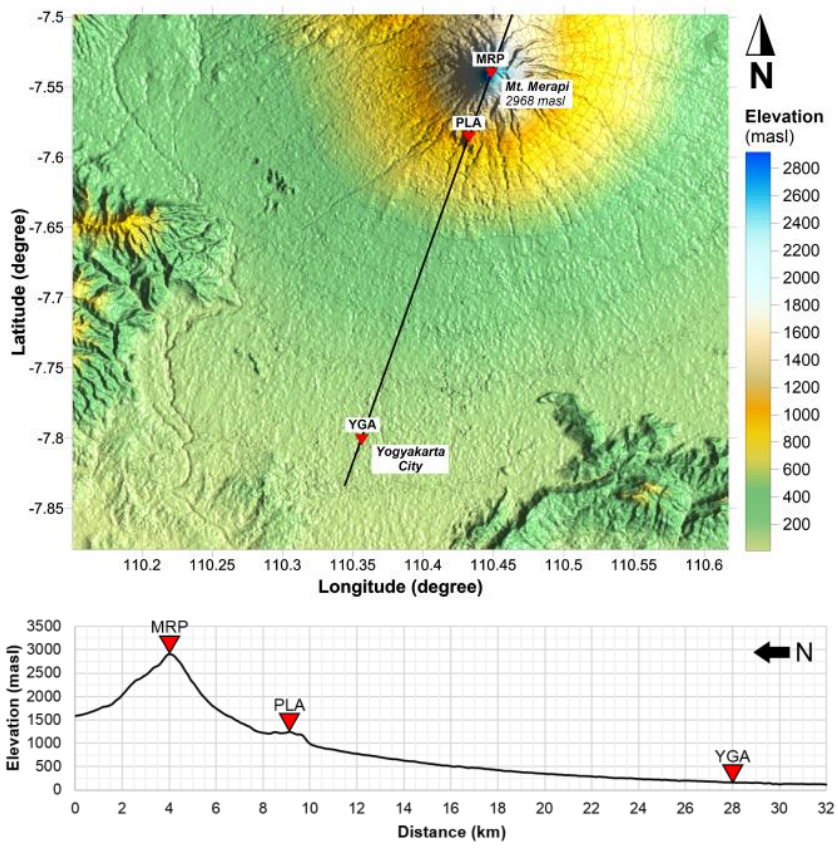

Figure 5. Selected line (black solid line) on the elevation map, from which the topography profile will be extracted. Three fictional station are shown in red triangle.

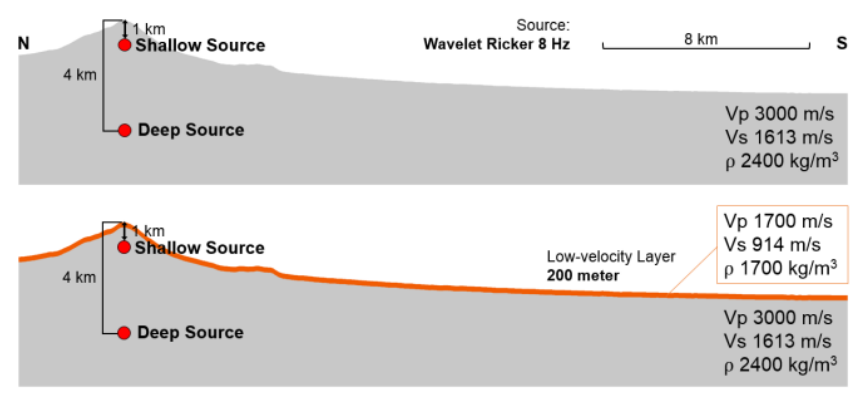

Figure 6. Homogeneous version of the medium (top) and the two-layer, heterogeneous version (bottom). area (YGA), which is refer to actual location of Yogyakarta city.

\section{B. Case Study of Mount Merapi}

There is two medium model created for this simulation, both is elastic medium and has similar free-surface topography (Fig. 6) and has dimension of 8000x1600 grid points, thus each grid point represents area of $4 \times 4$ meters $(\Delta x=\Delta z=4)$. The first medium is the homogeneous version, which has $\mathrm{P}$-wave velocity of $3000 \mathrm{~m} / \mathrm{s}$, and $V p / V s$ ratio is 1.86 [12]. The density value was based on [13] for Andesitic rock, is $2400 \mathrm{~kg} / \mathrm{m}^{3}$.

The second medium is the heterogeneous version similar to the first version, but at depth of 0-200 meter from topography has been replaced by low-velocity layer, with Pwave velocity of $1700 \mathrm{~m} / \mathrm{s}$ and density value of $1700 \mathrm{~kg} / \mathrm{m}^{3}$, based on density of alluvium and volcanic deposit of Merapi [19]. Both model will be used under the assumption that there is no attenuation occurs during the simulation.

The simulation will also performed using two scenarios, each with different source depth. In first scenario, the source is located at $4 \mathrm{~km}$ depth under the summit, where in other scenario, the source is set at $1 \mathrm{~km}$ depth. Both sources are 8 $\mathrm{Hz}$ point source of Ricker function. The sources depth and frequency are based on description of seismic activity of Merapi during 1983-1994 period by [12] for volcano tectonic event type-A (VTA, deep source scenario) and type-B (VTB, shallow source scenario).

\section{Numerical Modelling: CPU vs GPU}

The program used to numerically solved (1) is written in standard $\mathrm{C}++$ for computation using $\mathrm{CPU}$ and CUDA $\mathrm{C}++$ for parallel computation using GPU. Since GPU cannot directly perform file read/write, these routines, including medium parameters input and saving output seismogram and snapshots will performed by CPU in both program. Simulation for all scenarios are performed in both CPU and GPU, in order to compare the results. However, the results shown here are the results from computation using GPU only. Comparison between CPU versus GPU will described later at the discussion section.

\section{RESULT AND DISCUSSION}

In all four scenarios (each with different medium and source depth). The simulation was performed with 37500 step and $\Delta t$ of 0.0008 seconds to simulate seismic wave propagation in 30 seconds. The stability number is 0.8485 and is consistent with the stability conditions. The absorbing PML layer is 40 grid points thick, assigned at the left, right, and bottom edges of the medium. Dirichlet condition was applied to the outermost grid points [3], except for the upper boundary.

Seismograms from each scenario will be analyzed to identify the arrival time of P-wave, S-wave, and surface wave (SW). All seismograms are shown in normalized amplitude for both $\mathrm{X}$ - and $\mathrm{z}$-component. Snapshots of wave propagation in grayscale are shown at the end of this paper. 


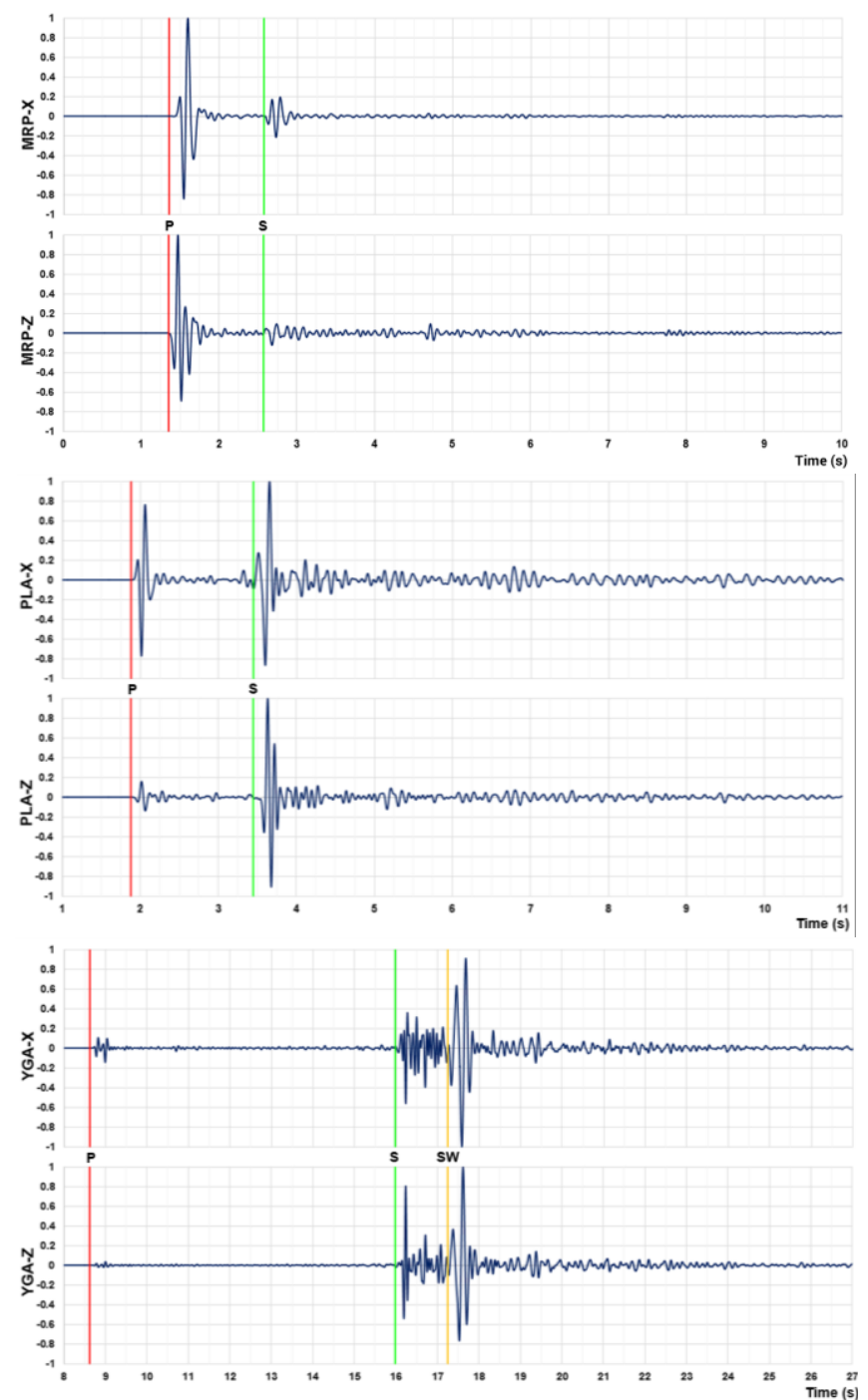

Figure 7. Synthetic seismogram from simulation with homogeneous model and deep source.

\section{A. Results With Homogeneous Model}

Result from simulation using deep seismic source is described as follows. In the model, MRP station is located directly above the source, thus the recorded wave came directly from the source point, and the body waves arrival is clearly identified in the seismogram (Fig. 7). Reflected waves from free-surface are also visible in the snapshot.

Body waves were clearly observed at PLA. At this distance, there is no surface wave observed in the seismogram. From the snapshots, the surface wave seems to generated after $t=5$ seconds at further distance from the source than PLA position.

At YGA, surface wave is clearly identified with higher amplitude than recorded body waves. There is "fake" P-wave recorded shortly after actual P-wave arrival. This unwanted wave is reflected wave from PML layer, indicated that wave energy was not perfectly absorbed along the boundary. Thus,

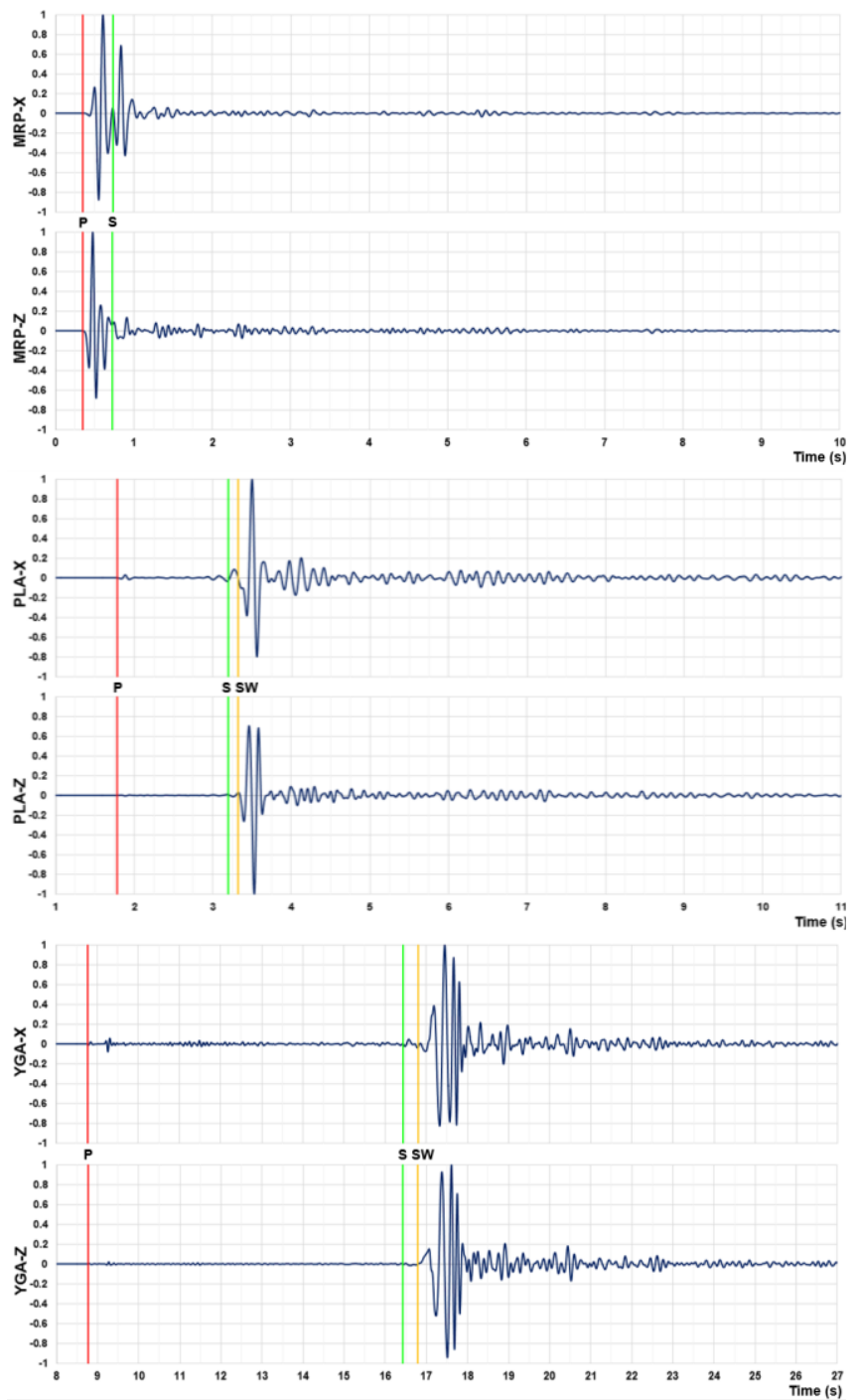

Figure 8. Synthetic seismogram from simulation with homogeneous model and shallow source.

the actual P-wave arrival, which amplitude is very small, was determined by observing the snapshots.

When using shallow seismic source, body waves arrival at MRP are recorded early compared to simulation using deep source, which is obviously caused by closer source location to the station. The offset between P-wave and Swave arrival became smaller, which made it difficult to separate the S-wave arrival in the seismogram (Fig. 8).

At PLA, the body waves arrival are recorded at approximately same time compared to deep source simulation, despite the closer source location to the station. This might be caused by the presence of topography between the source and the station, which forced the seismic waves to travelled along the medium surface and propagated through longer path to the station. The presence of topography also caused surface wave to be generated early. The surface wave arrival at PLA were recorded shortly after S-wave arrival with much higher amplitude, dominating the body waves amplitude. Similar condition are also observed in YGA. 


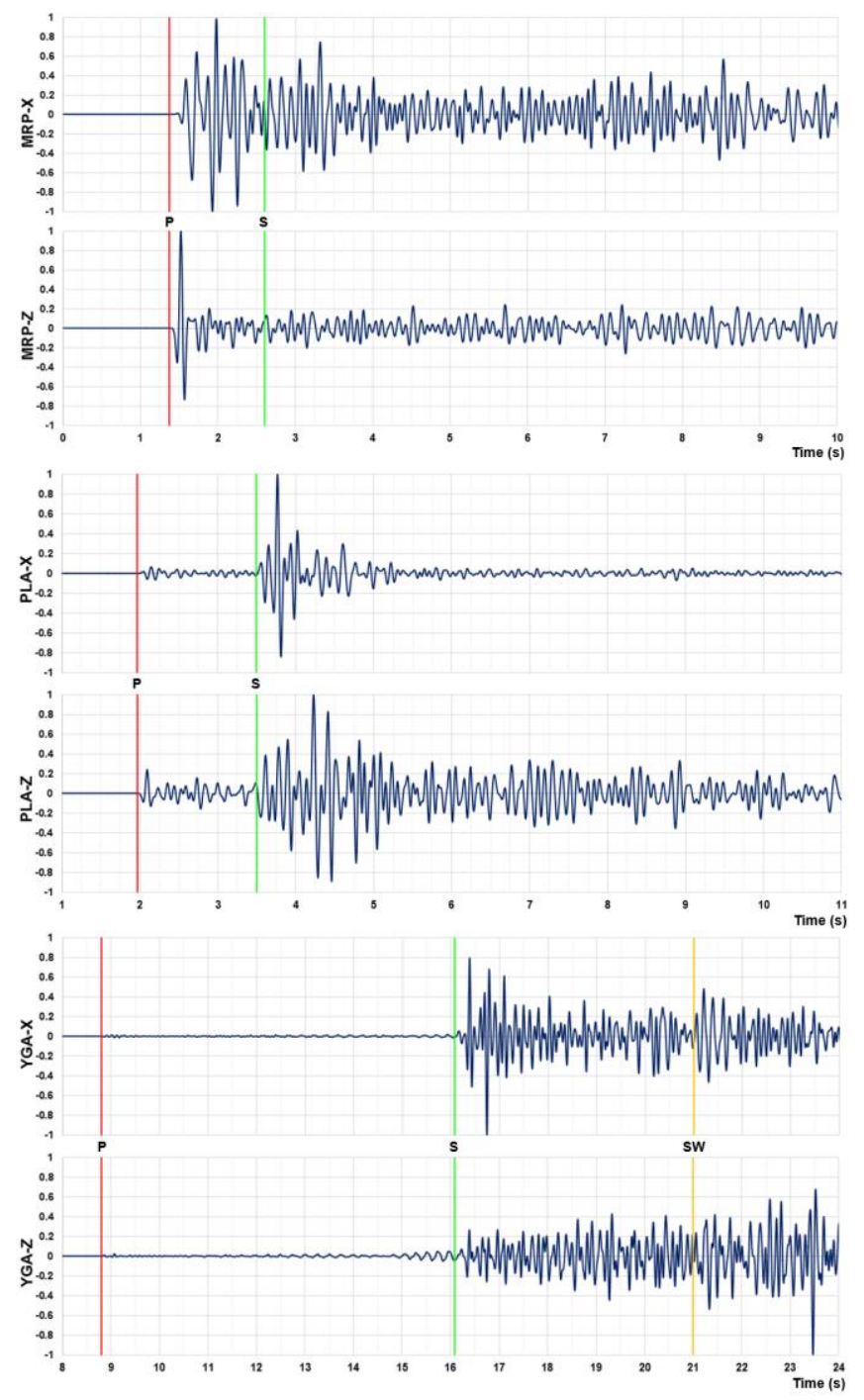

Figure 9. Synthetic seismogram from simulation with heterogeneous model and deep source.

\section{B. Results With Heterogeneous Model}

Synthetic seismograms obtained in this simulation (and next simulation) are much more complex compared to previous simulation. This complexity suspected to be caused by multiple reflection within low-velocity layer, and scattering of surface wave. The overall characteristics of body waves and surface wave arrival are similar to previous scenarios, although the complexity of the seismograms made it more difficult to identify the arrival of main phases of the waves. When using deep source, seismogram at MRP and PLA are still clearly showing the arrival of body waves, while seismogram at YGA is more "deceiving": The P-wave arrival is extremely small to observed, and surface wave arrival are difficult to identified (Fig. 9).

By using shallow source, at MRP it is visually impossible to separate the S-wave arrival from recorded P-wave. In this study, the identification has made easier by observing the snapshots. At PLA, the amplitude changes more gradually

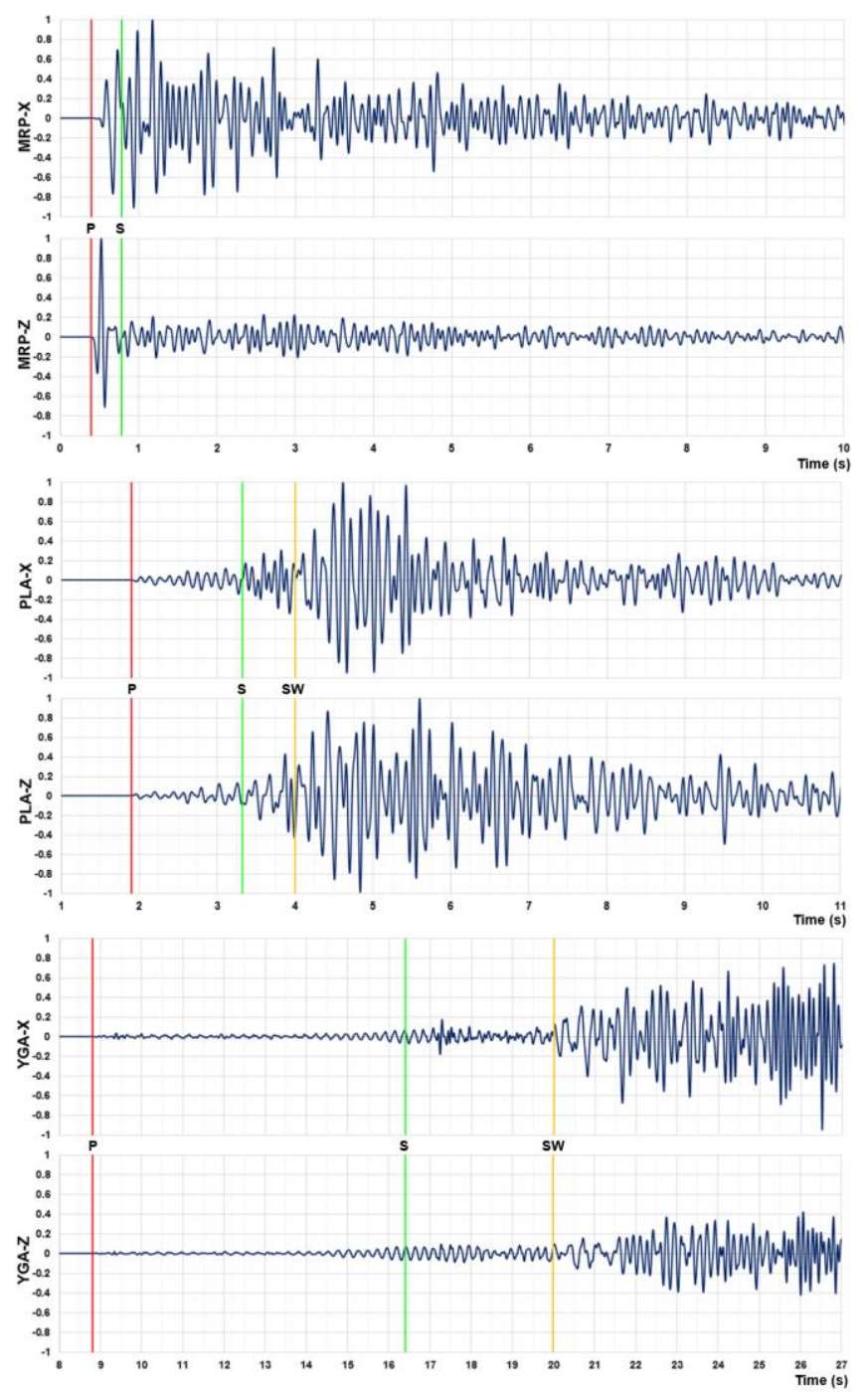

Figure 10. Synthetic seismogram from simulation with heterogeneous model and shallow source.

between wave phases arrival. This might be caused by multiple reflection within low-velocity layer, which became more intensive with increasing distance. At YGA, the surface wave has visually dominating the seismogram, while the body waves amplitude is extremely small to observed (Fig. 10).

\section{Frequency Analysis of The Seismogram}

If the medium is designed to mimic a infinite homogeneous medium by assigning PML layer on all edges of the medium, then one could assume that in any points with constant distance from the source, the frequency versus time plot (spectrogram) of the waves recorded at that point would be similar, unless there is discontinuities or heterogeneity in the medium, like the one used in this study. Fig. 11 are the spectrograms from simulation in homogeneous layer using deep source, where the wave phases arrival could be clearly identified. P-wave phase has dominant frequency of 7-10 Hz, $\mathrm{S}$-wave has higher frequency value of $5-15 \mathrm{~Hz}$, although the 

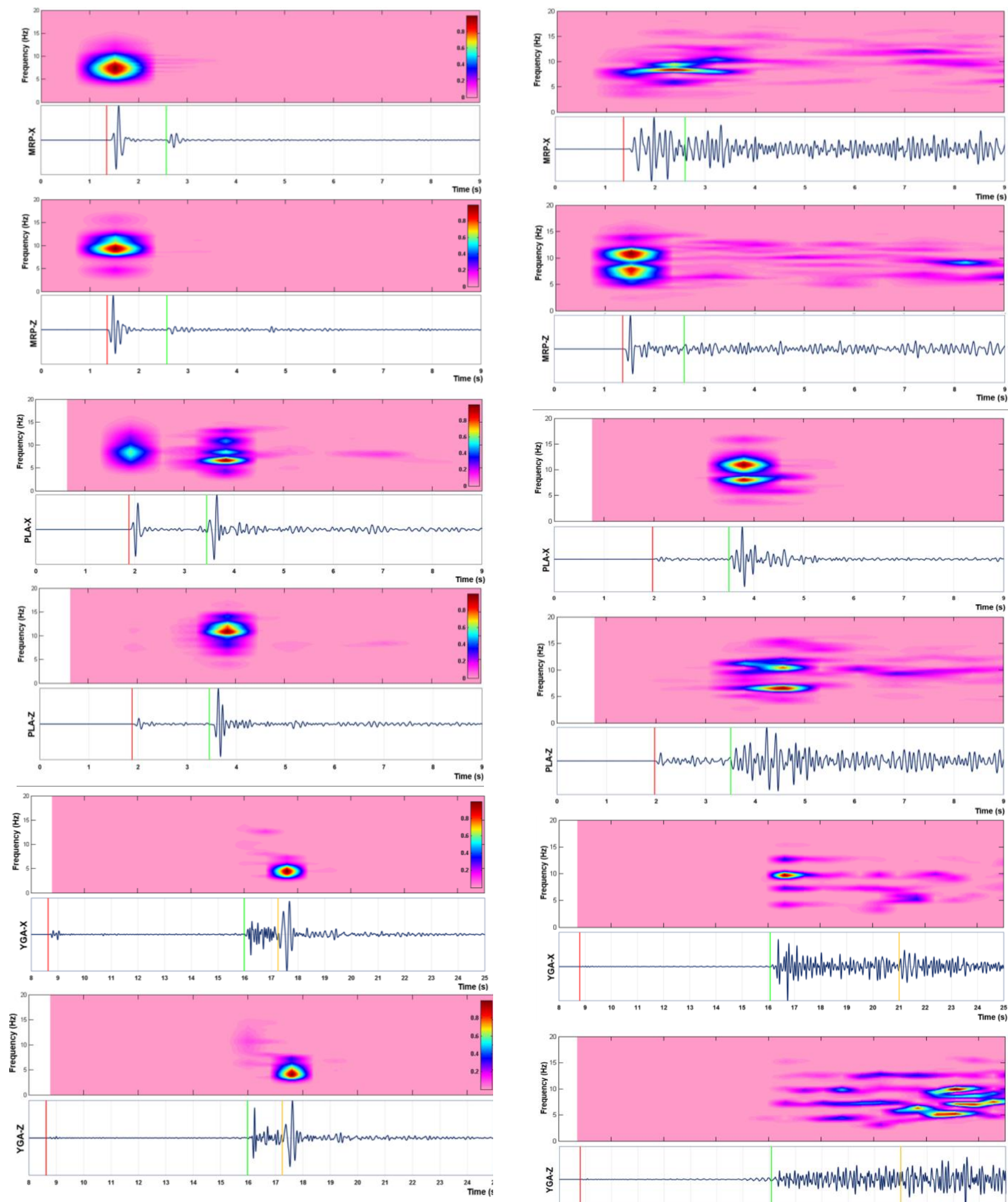

Figure 11. Spektrogram from simulation in homogeneous medium using deep source. Color scale represents normalized density.
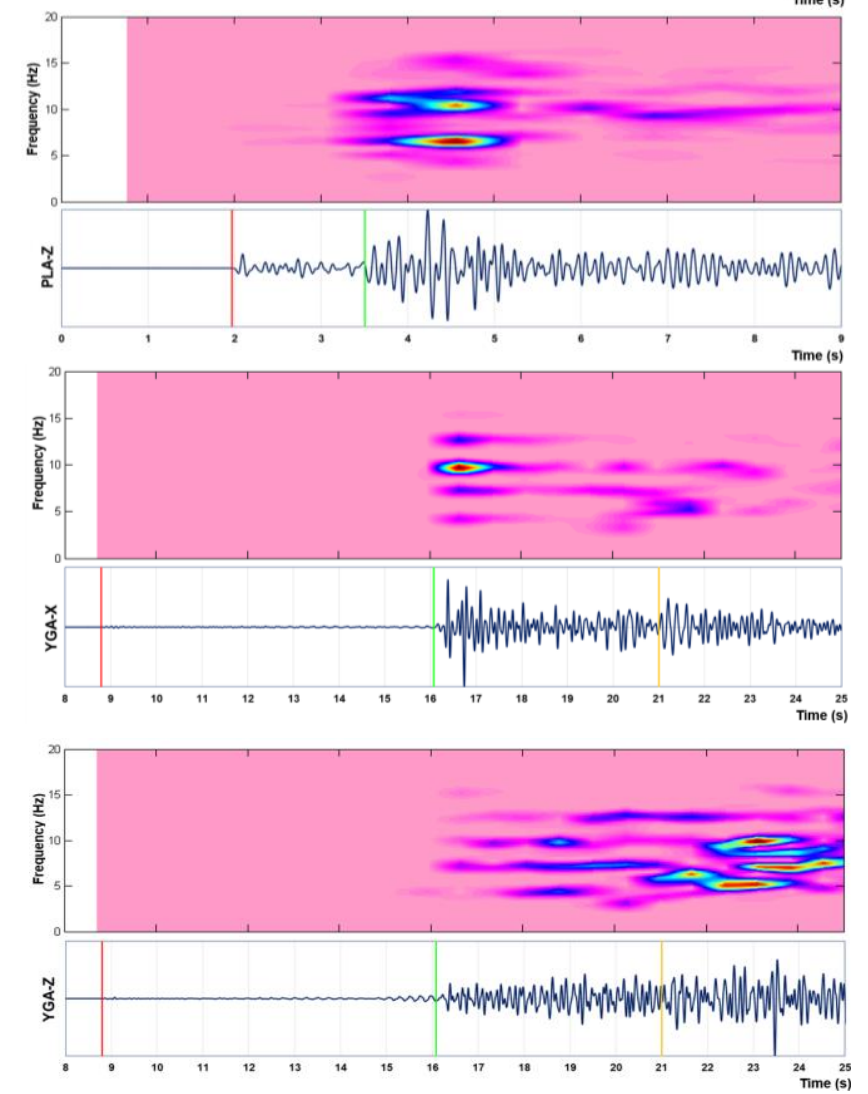

Figure 12. Spektrogram from simulation in two-layer, heterogeneous medium using deep source. 
dominant frequency is relatively higher than $\mathrm{P}$-wave frequency. Surface wave (SW) has lower frequency range of 2-8 Hz.

Fig. 12 is the spectrograms from simulation in two-layer medium using deep source. P-wave phase has dominant frequency of 5-12 Hz, best seen in MRP station, where Pwave is dominant in the seismogram. S-wave has wider frequency range of 4-15 Hz, although its dominant frequency is relatively higher than $\mathrm{P}$-wave frequency. Surface wave has higher frequency range of 7-10 Hz (at PLA) and 5-6 Hz (at YGA), compared to simulation in homogeneous medium.

From the spectrograms, we could observed that there is multiple values of high frequency density at single time value. It is suspected to be caused by mutual interference of multiple reflected waves in low-velocity layer. The reflection occurred at the bottom interface of low-velocity layer, and at the topography.

\section{Inaccuracies And Instability of the Simulation}

The simulation results, however, still affected by the inaccuracies and instability of the simulation technique. There is "tail" characteristic of surface waves observed in the seismogram, which might be caused by numerical dispersion, or by separation of velocity components in staggered-grid scheme, where $v_{x}$ and $v_{z}$ are actually separated by 2.8 meters [1].

Seismograms from simulation in multi-layer medium shows that the end of an event (coda) was difficult to determined. The presence of low-velocity layer might be responsible for the complex coda [20]. In [1], a simulation using irregular free-surface and flat bottom interface has been performed, and found that topography could cause seismic energy scattering.

Explanation about what caused the disappearance of Swave arrival in $\mathrm{z}$-component seismogram from MRP is not clear, since it is not observed during simulation using CPU. It might be caused by grid-points classification along the free-surface used by Robertsson's method which, when combined with GPU's parallel computation scheme, accidentally nulls the velocity value.

The generated surface wave is identified as Rayleigh wave [21] stated that Rayleigh wave energy is getting stronger if the source is located closer to the free-surface, which explains the difference of surface wave between the usage of deep seismic source and shallow seismic source.

While performing simulation in heterogeneous medium, numerical oscillation has occurred at time step larger than 22 seconds (Fig. 13). This oscillation is not observed during simulation using CPU, and tend to spread to almost the entire medium as the simulation progressed. This suspected to be caused by single-precision computation accuracy, finitedifference accuracy, which is fourth order spatial operator, decreasing accuracy along topography, and ineffective thread synchronization in GPU device, since one cannot knowing which thread will be processed by the GPU.

To compare the seismogram obtained from simulation using CPU versus simulation using GPU, we have performed a simulation of seismic wave propagation in two homogeneous medium, first medium has flat free-surface,
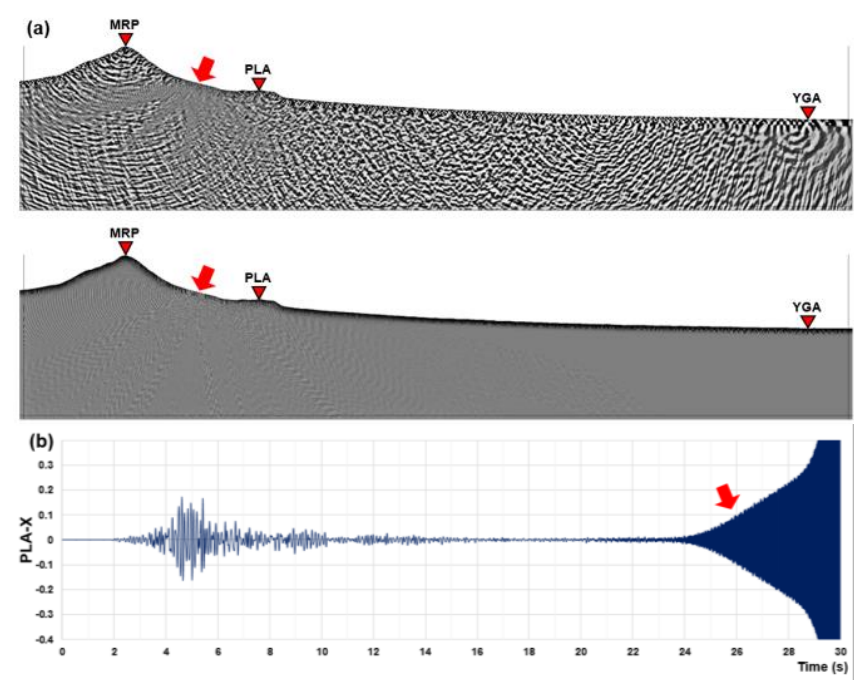

Figure 13. (a) Snapshots at $t=20.8 \mathrm{~s}$ and $t=29.9 \mathrm{~s}$. Red arrow shows the location where numerical oscillation started. The grayscale color scale was edited to clearly shows this unwanted oscillation. (b) Seismogram from PLA station affected by the oscillation.

(a)
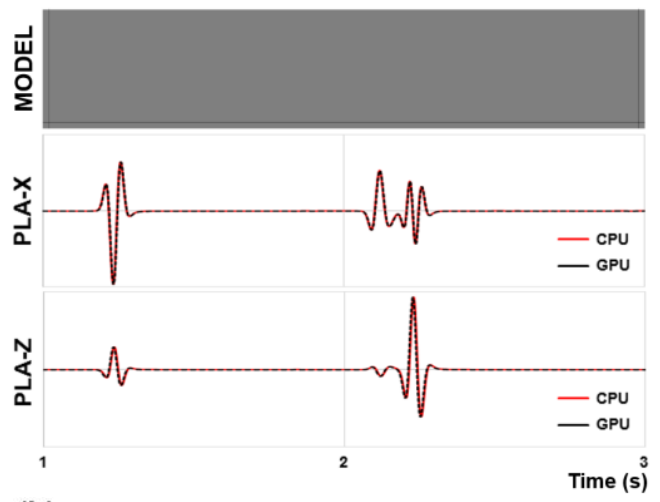

(b)
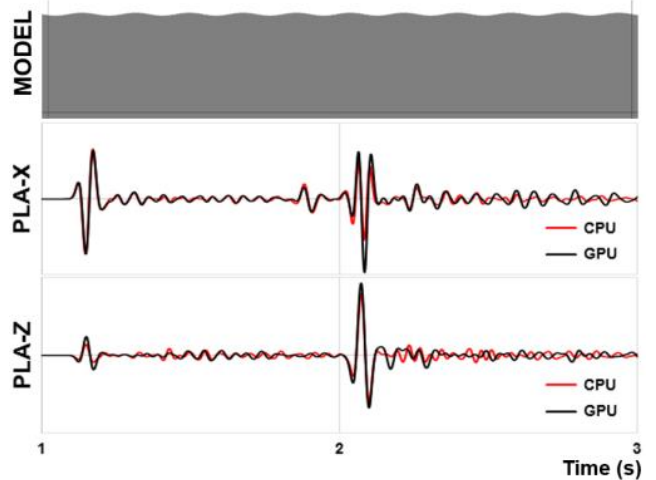

Figure 14. (a) Comparison of seismogram obtained from simulation using CPU and GPU with simple homogeneous model. The black line here is shown dashed to show the red line beneath it. (b) Results using model with simple topography, showing different waveform between using CPU and GPU. 
Heterogeneous Medium: Shallow Source (23.66x Acceleration)

$\begin{array}{rrrrrrrrr}0 & 200 & 400 & 600 & 800 & 1000 & 1200 & 1400 & 1600 \\ & & & & & & & & \end{array}$

Figure 15. Comparison of total execution time between using CPU (blue bar) versus using GPU (green bar) and the time acceleration value for each scenario in this study.

while the other medium has simple, sinusoidal-like topography (Fig. 14). Medium with flat free-surface resulted in perfectly matched seismogram by using either CPU or GPU. But different result was obtained while using second medium with simple topography. There was phase difference observed after S-wave arrival, although body wave arrival was correctly modelled.

This phase difference, specifically in highly detailed wave might be caused by grid dispersion [2]. Although, in this study, amplitude difference is more likely caused by difference in computation concept of CPU versus GPU, where the CPU tend to process the data sequentially, while to GPU process the data in parallel.

Thus it could be concluded that Robertsson method [1] to handle the free-surface in this study is still has its weaknesses when directly applied to GPU's parallel computation scheme, although there is still possibility that this method will be successfully applied to parallel computation scheme.

\section{E. Execution Time Comparison: $C P U$ vs GPU}

This comparison will be focused to GPU's capability to reduced execution time while compared to the CPU. The execution time will be defined as the time required to perform time iteration, including calculation and update of velocity and stress components, source and boundary condition calculation, and saving the seismogram and snapshots. The time spent for loading medium parameters, preparing and initialization of parameters, including PML layer and damping parameter, are not taken into account, since those routines are always performed using CPU in local memory.

All simulation in this study was performed using desktop computer with an Intel Core i7 processor, 32 GB RAM and an NVIDIA GeForce GTX 590 graphics card with 1.5 GB memory and compute capability of 2.0. The simulation program was designed with single-precision accuracy.

The comparison results are shown in Fig. 15. Usage of GPU has proved to be able to accelerate the execution by 23.24 times in homogeneous medium, and 23.66 times in heterogeneous, two-layer medium. Better results can be achieved by using newer version of the graphics card.

\section{CONCLUSION}

We have performed a series of simulation of seismic wave propagation using fourth order finite-difference method in elastic medium with presence of topography of Mount Merapi, Yogyakarta. The results has been presented in seismograms and snapshots, showing significantly different results from four scenarios with two medium condition (with and without low-velocity layer) and two different source depth.

The usage of GPU has been successfully accelerate the computation time by 23.24 times in homogeneous medium and 23.66 times in heterogeneous medium, compared to using only CPU. Better results can be achieved by using newer graphics card, or by using multi-GPU.

However, this study focused only on very simple case of earth medium. Better, more realistic results may be achieved if the simulation was designed to handle more realistic earth medium, such as the presence of fault system or groundwater layer.

\section{REFERENCES}

[1] Robertsson, J. O. A., "A numerical free-surface condition for elastic/viscoelastic finite-difference modeling in the presence of topography," Geophysics, Vol. 61, pp. 1921-1934, NovemberDecember 1996.

[2] Levander, A. R., "Fourth-order finite-difference P-SV seismogram," Geophysics, Vol. 53, pp. 1425-1436, November 1988.

[3] Komatitsch, D., and Martin, R., "An unsplit convolutional Perfectly Matched Layer improved at grazing incidence for the seismic wave equation," Geophysics, Vol. 72, pp. SM155-SM167, SeptemberOctober 2007, doi:10.1191/1.2757586.

[4] Michéa, D., and Komatitsch, D., "Accelerating a 3D finite-difference wave propagation code using GPU graphics cards," Geophys. J. Int., Vol. 182, pp. 389-402, November 2010, doi: 10.1111/j.1365246X.2010.04616.x

[5] Webb, C. J., "Computing 3D Finite Difference schemes for acoustics - a CUDA approach," Dissertation, MSc in Accoustics and Music Technology, University of Edinburgh, August 2010, unpublished.

[6] Kirk, D. B., and Hwu, W. W., Programming Massively Parallel Processors: A Hands-on Approach, Burlington, MA: Elsevier, 2010.

[7] Virieux, J., "SH-wave propagation in heterogeneous media: Velocitystress finite-difference method," Geophysics, Vol. 49, pp. 1933-1957, November 1984.

[8] Virieux, J., "P-SV wave propagation in heterogeneous media: Velocity-stress finite-difference method," Geophysics, Vol. 51, pp. 889-901, April 1986

[9] Madariaga, R., "Dynamics of an expanding circular fault," Bull. Seism. Soc. Am., Vol. 66, pp. 639-666, June 1976.

[10] Shearer, P. M., Introduction to Seismology, 2nd ed., New York: Cambridge University Press, 2009.

[11] Gertisser, R., et al, "Merapi (Java, Indonesia): anatomy of a killer volcano," Geology Today, Vol. 27, March-April 2011, pp. 57-62, doi: 10.1111/j.1365-2451.2011.00786.x

[12] Ratdomopurbo, A., and Poupinet ,G., "An overview of the seismicity of Merapi volcano (Java, Indonesia), 1983-1994," Journal of Volcanology and Geothermal Research, Vol. 100, pp. 193-214, 2000.

[13] Telford, W. M., Geldart, L. P., and Sheriff, R. E., Applied Geophysics, 2nd ed., New York: Cambridge University Press, 1990.

[14] Roden, J. A., and Gedney, S. D., "Convolutional PML (CPML): An Efficient FDTD Implementation of the CFS-PML for Arbitrary 
Media," Microwave and OpticalTechnology Letters, Vol. 27, pp. 334-339. June 2000,

[15] Bérenger, J. P., "A perfectly matched layer for the absorption of electromagnetic waves," Journal of Computational Physics, Vol. 114, pp. 185-200, 1994.

[16] Bamberger, A., Chavent, G., and Lailly, P., "Etude de schémas numériques pour les équations de l'élastodynamique linéaire," Rapports de recherché, No. 41, INRIA, October 1980.

[17] Fuyuki, M., and Matsumoto, Y., "Finite-difference analysis of Rayleigh wave scattering at a trench," Bull. Seis. Soc. Am., Vol. 70, pp. 2051-2069, 1980

[18] Sanders, J., and Kandrot, E., CUDA by Example: An Introduction to General Purpose Programming, USA: NVIDIA Corporation, 2010.

[19] Ridhwan, M., "Interpretasi Struktur Bawah Permukaan Daerah Kretek dan Sekitarnya, Kabupaten Bantul Provinsi D.I. Yogyakarta Berdasarkan Data Gravitasi," Thesis, Department of Physics, Universitas Diponegoro, 2010, unpublished.

[20] Levander, A. R., and Hill, N. R., "P-SV resonance in irregular lowvelocity surface layer,” Bull. Seis. Soc. Am., Vol. 75, pp. 847-864, 1985.

[21] Zhou., Z. S., Liu, X. L., and Xiong, X. Y., "Finite-difference modelling of Rayleigh surface wave in elastic media," Chinese J. Geophys., Vol. 50, pp. 496-504, 2007.

\section{SNAPSHOTS OF WAVE PROPAGATION}

(velocity components in grayscale color, from dark color for negative value and light color for positive value.

$\mathrm{U}$ denotes north and $\mathrm{S}$ denotes south)

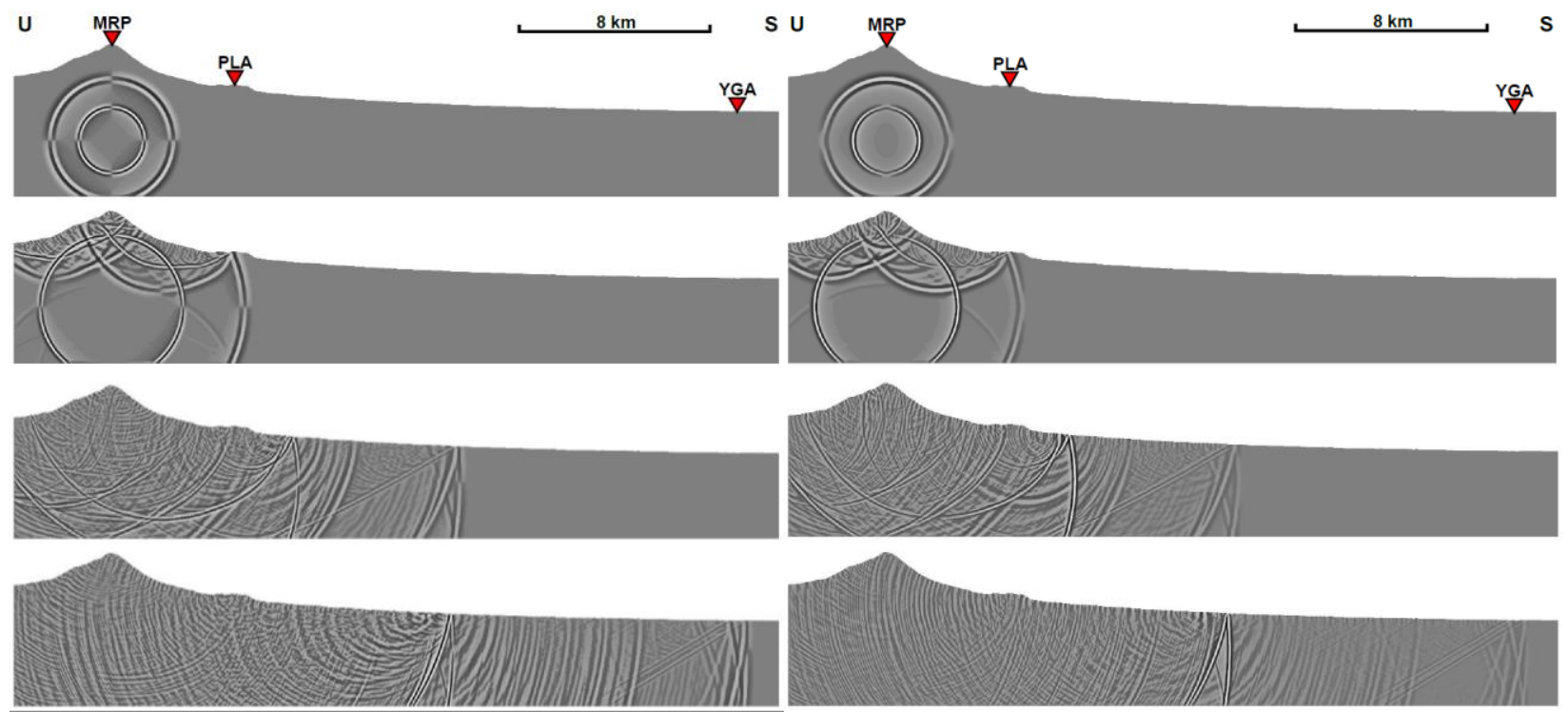

Figure 16. Snapshot from homogeneous medium-deep source scenario at $t=1, t=2, t=5$, and $t=9$ from (left) $v_{x}$-component and (right) $v_{z}$-component. 


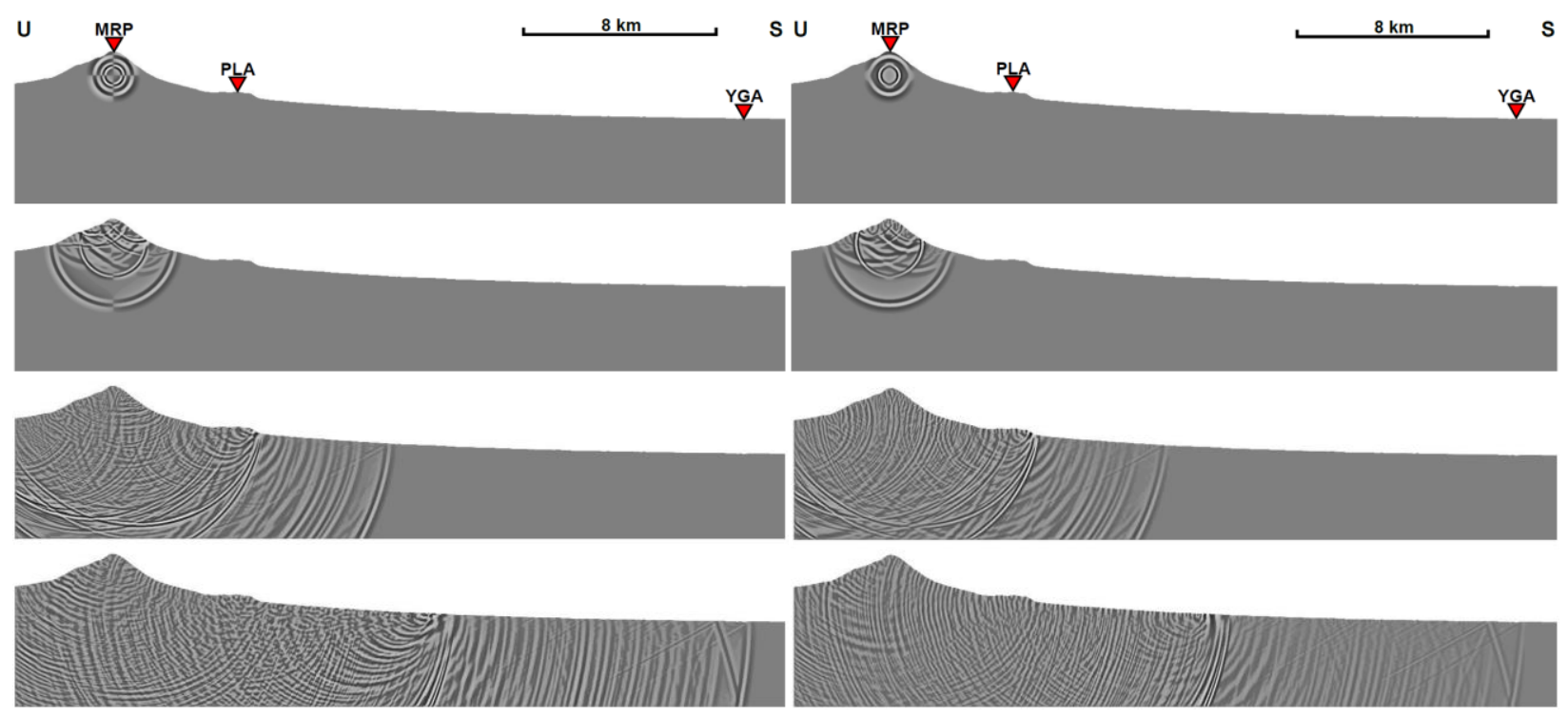

Figure 17. Snapshot homogeneous medium-shallow source scenario at $t=0.4, t=1, t=4$, and $t=9$ from (left) $v_{x}$-component and (right) $v_{z}$-component.
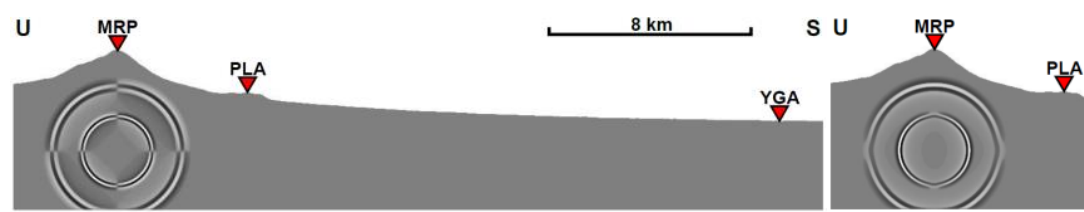

$\mathrm{km}$

s
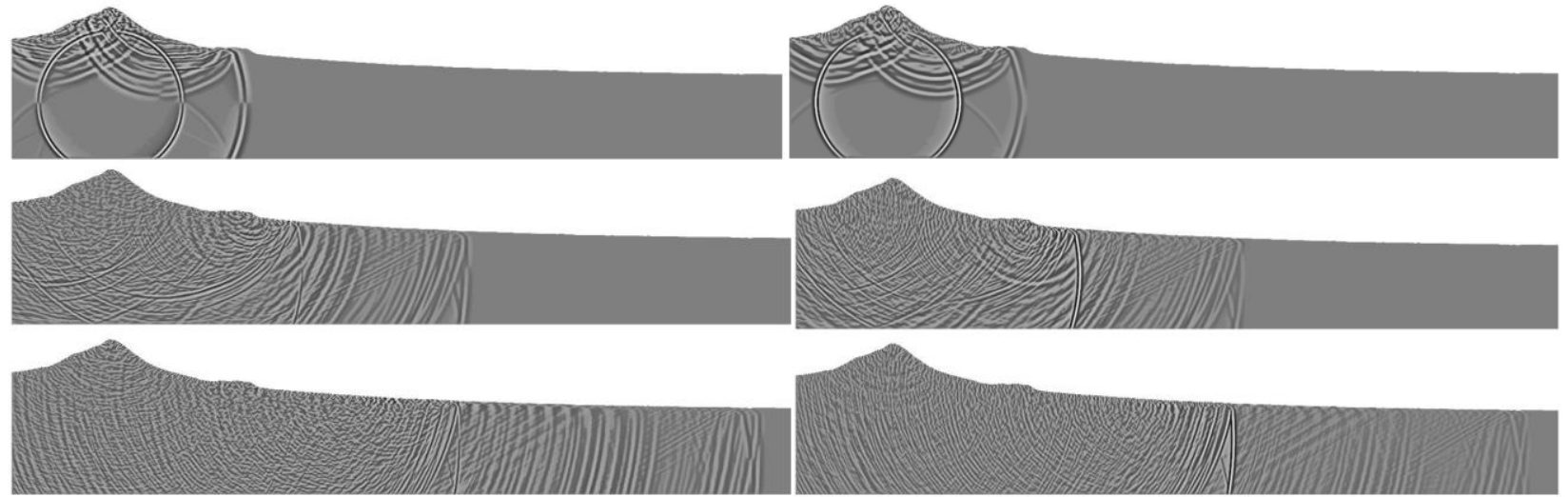

Figure 18. Snapshot from heterogeneous medium-deep source scenario at $t=1, t=2, t=5$, and $t=9$ from (left) $v_{x}$-component and (right) $v_{z}$-component 


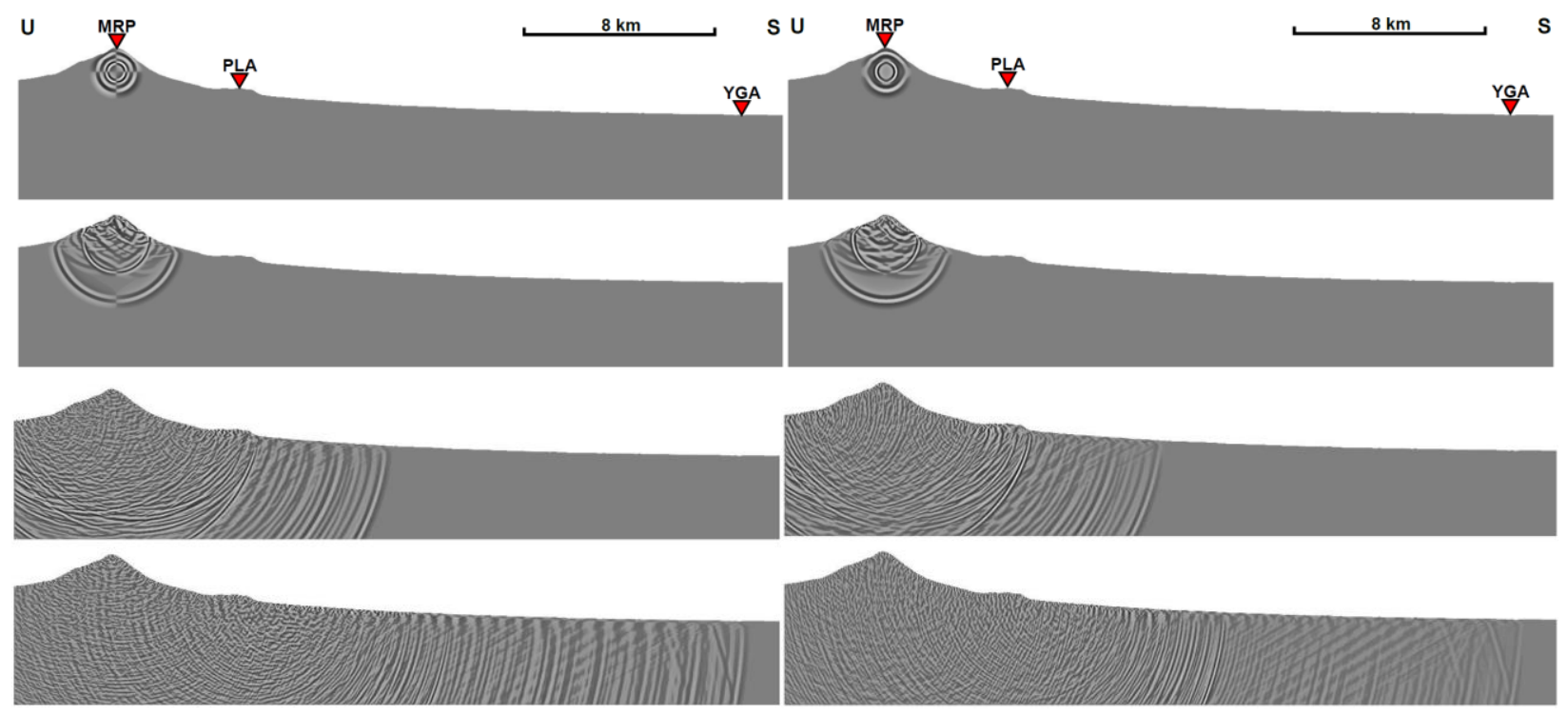

Figure 19. Snapshot heterogeneous medium-shallow source scenario at $t=0.4, t=1, t=4$, and $t=9$ from (left) $v_{x}$-component and (right) $v_{z}$-component. 